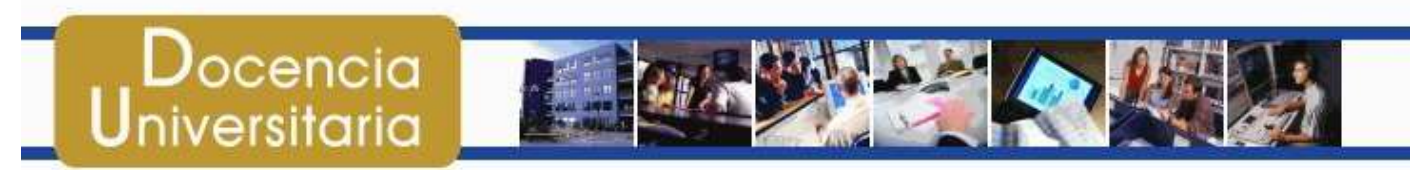

Revista Digital de Investigación en Dacencia Universitaria / Añ̃ 3 - ND1- Jun. 2007

\title{
El Aspecto Lúdico del e-Learning: El juego en entornos virtuales de aprendizaje
}

\author{
Lucio Margulis
}

\section{Resumen}

El uso de las tecnologías de la información y la comunicación (TICs) está revolucionando la forma de aprender, enseñar y jugar de las nuevas generaciones y las organizaciones modernas. Incluso, podemos apreciar cómo -en muchos casos- las personas aprenden más gracias al entorno (compañeros, situación de aprendizaje, elementos e institución con la que interactúan), que por los contenidos específicos o el docente del curso. En este artículo propongo compartir una mirada particular sobre el aspecto lúdico de la producción de contenidos multimediales para proyectos de educación basados en modalidades de "e-Learning" (aprendizaje electrónico) y "Blended Learning" (formato mixto que combina lo mejor del mundo real y del virtual).

\section{Palabras clave: e-learning, entornos virtuales, juegos virtuales, realidad virtual}

\begin{abstract}
Abastract
Playful aspects of e-learning: the game in virtual learning surroundings

The use of 'Information and Communication Technologies' (ICT's) is revolutionizing the way of learning, teaching and playing of new generations and modern organizations. We can appreciate how in many cases people learn more due to their surroundings (classmates, learning situations, elements and institutions with which they interact), than because of the specific course teacher.

In this article I propose we share a particular perspective concerning the playful aspect of the multimedia contents for education projects based on 'e-learning' (electronic learning) and 'Blended Learning' (mixed format that combines the best of the real and virtual world).
\end{abstract}

Key words: e-learning, virtual surroundings, learning games, virtual reality. 


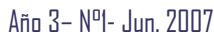

\section{Introducción}

El aprendizaje puede tornarse un proceso aburrido, falto de elementos motivadores y emocionales. En la educación a distancia, esta dificultad es mayor, debido a la soledad inicial del alumno frente a la computadora y la dificultad de reemplazar el aprendizaje cara a cara por el aprendizaje virtual. Una complicación que ha llevado al e-learning del mismo modo que a otras modalidades de educación a distancia- a sufrir un alto grado de deserción de los alumnos por falta de motivación y de compromiso con los otros seres virtuales. Por estos motivos, trataré de demostrar que el juego multimedial, combinado con el cooperativo, constituyen importantes estrategias en estas modalidades.

En efecto, las actividades lúdicas pueden ayudar al e-learning a motivar al alumno y retenerlo hasta que complete sus estudios:

1. El juego multimedia ayuda a los estudiantes a disfrutar de un momento placentero, participativo, interactivo y desafiante frente a la computadora. Promueve la experimentación y nos enfrenta inicialmente a problemas virtuales, pudiendo cometer errores y aprender de ellos sin consecuencias directas sobre la realidad.

2. El juego cooperativo, en cambio, permite a los alumnos establecer una relación más estrecha con sus compañeros, debido a su capacidad integradora.

A lo largo de este artículo, presentaremos inicialmente algunas ideas sobre el aspecto lúdico en el aprendizaje, para luego tratar de establecer la relación entre el e-learning y el juego. Continuaremos proponiendo un viaje por el mundo de las dinámicas multimediales y cooperativas de simulación, para, finalmente, analizar la manera de trasladar las actividades lúdicas cara a cara a entornos virtuales de aprendizaje, mostrando las ventajas que brinda su utilización.

En este texto, los lectores encontrarán un juego oculto, que invitamos a descubrir, y cuya solución encontrarán al final. 
Añ̃ 3 - ND1- Jun. 2007

\section{El Juego y el Aprendizaje}

El hombre ha aprendido siempre por medio del juego. El mejor ejemplo está en los niños, que conocen el mundo jugando desde su nacimiento. En el jardín de infantes, las actividades lúdicas constituyen un recurso fundamental para el aprendizaje, aunque en el resto de su escolaridad parecieran destinadas a ocupar exclusivamente el espacio de los recreos o de algunas materias específicas. Como decía Pablo Neruda, "el niño que no juega no es niño, pero el hombre que no juega ha perdido al niño que habita en él y que le hará mucha falta”.

Es preciso aclarar que en este texto me basaré en el planteamiento que realiza Johan Huizinga en su libro "Homo Ludens", donde expresa que "el juego, en su aspecto formal, es una acción libre ejecutada 'como si', y sentida como situada fuera de la vida corriente, pero que, a pesar de todo, puede absorber por completo al jugador, sin que haya en ella ningún interés material".

En efecto, las actividades lúdicas han sido unas de las modalidades más efectivas de aprendizaje desde la aparición del hombre en el mundo. A través de ellas es posible aprender diferentes cosas, algunas placenteras, otras conflictivas, como experimentar, equivocarse y ponerse en el lugar de otros.

Para la pedagogía, el juego es una de las más ricas estrategias de simulación, mientras que en el ámbito empresarial, y sobre todo a partir del surgimiento de la teoría de la inteligencia emocional, las actividades lúdicas han comenzado a utilizarse con el fin de trabajar los aspectos emotivos de las personas dentro de las organizaciones.

"El juego es una manera de aprendizaje de vida. Los niños/as juegan a simular los roles de los mayores, mientras que los mayores juegan para ensayar sus actitudes sin riesgo real. Así los juegos hacen cambiar de rol por unos instantes y ponen a prueba los conocimientos y destrezas de los jugadores", como señala el estudio "Juego y la Inteligencia Emocional”, de Tareixa Barberena.

\section{El e-Learning y el Juego}

El e-Learning nos brinda la posibilidad de convertirnos en verdaderos protagonistas de nuestro proceso de aprendizaje, mientras que la tecnología nos ofrece -entre otras cosas- 


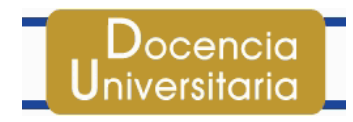

Aก๊̃ 3 - Nㅏ- Jun. 2007

la velocidad, la interactividad y la posibilidad de colaborar e interactuar con otros, a través de diversas herramientas y canales. La experiencia de navegar en Internet, recorrer hipertextos, enviar e-mails, y chatear, es -justamente- un juego en sí mismo: un viaje o $\underline{\text { una }}$ aventura que emprendemos, tanto los consumidores como los productores de este mundo virtual.

La relación entre e-Learning y juego se inicia en la dinámica lúdica planteada por la navegación a través de hipertextos. "Las características determinantes del fenómeno lúdico (la libertad, la dualidad, el espacio y tiempo propios, el automovimiento, el azar y la doble productividad) se relacionan con el "libro-juego" en general, y con la narrativa hipertextual en particular, en tanto este nuevo medio de lectura y escritura promueve la libertad creativa, la reconfiguración del autor y del lector, el espacio festivo y virtual, la navegación infinita, la mediación del azar y la necesidad y la interactividad", señala Marcela Castro en su artículo "Juego e Hipertexto". (Pontificia Universidad Javeriana, de Colombia).

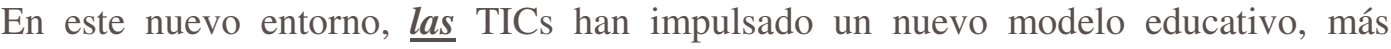
participativo, interactivo, ameno y colaborador, en el que el juego recobra su protagonismo y puede contribuir a la transformación de la educación en una actividad más entretenida e interesante.

\section{Tipos de Juegos en E-Learning}

A continuación presentaremos ante $\underline{\text { usted }}$ es una posible clasificación de los juegos que se ofrecen en los entornos virtuales de aprendizaje: los que están basados en soluciones multimedia (animaciones, simulaciones y experiencias de realidad virtual), y aquellos utilizados para promover el conocimiento, la integración y la creación, que llamaremos cooperativos, ya que también ayudan a los tutores a conformar, mantener y fortalecer las comunidades de aprendizaje.

\section{Juegos Multimediales}

Los juegos multimediales simulan mundos, situaciones y seres, dándonos la oportunidad de sumergirnos en entornos cada vez más complejos y motivadores. Ni las simulaciones ni la realidad virtual son representantes de los nuevos recursos lúdicos de la multimedia; de hecho, los pilotos y los soldados se entrenan desde hace mucho tiempo en simuladores de vuelo y guerra, respectivamente. La gran diferencia producida en últimos años consiste en la enorme difusión y el uso masivo de este tipo de tecnologías. Un fenómeno derivado de esto es la creciente presencia de computadoras 
en los hogares, la multiplicación de los cybercafés y locutorios, y el uso masivo de los juegos para PC's y consolas (de tipo Play Station).

El perfeccionamiento de los sistemas y del software para simular entornos contribuyó a darle mayor realismo a los mismos. Así, en la actualidad las nuevas generaciones pasan más horas jugando que estudiando, y se desenvuelven mejor en el mundo virtual que en el real. Internet ha promovido la formación de comunidades de niños, jóvenes y adultos unidos tanto por los juegos multimediales como por los de roles. Un ejemplo interesante es la venta de los juegos de películas como Harry Potter, La Guerra de las Galaxias o El Señor de los Anillos, cuyas ganancias ya han superado a las cifras obtenidas por su exhibición en los cines. O bien, la adaptación al cine de las aventuras de personajes virtuales, como Mortal Kombat y Lara Croft.

Como sostuvo Antonio Moar, director general del grupo Moar, durante su ponencia sobre simulaciones en Online Educa Madrid 2004, las tendencias mencionadas dan cuenta de "la necesidad que tiene la gente de ser protagonista", de participar activamente y de $\underline{\text { modo }}$ placentero de experiencias concretas que les permitan poner a prueba sus conocimientos en entornos virtuales antes de enfrentarse a los reales. En definitiva, al igual que en los juegos multimediales, los alumnos desean ser actores protagónicos de su propio proceso de aprendizaje.

Quizás ha llegado el momento de pensar qué estamos haciendo los pedagogos, los diseñadores gráficos y multimediales, y los tutores para incorporar el juego, en sus distintas expresiones, a los programas educativos. En este sentido, la gran pregunta sería: ¿cómo podemos aprovechar las ventajas de las actividades lúdicas en el proceso de aprendizaje que se da en los entornos virtuales?

Posiblemente haya que observar lo que están haciendo a nivel mundial la mayoría de universidades, escuelas de negocios y corporaciones, que han incorporado los juegos de simulación de negocios individuales, grupales e interinstitucionales con el objetivo de poner a prueba las competencias de management de sus alumnos en la resolución de las situaciones más complejas y cambiantes del mercado internacional.

Podemos encontrar diversos tipos de simulaciones en el mercado:

- Simulación de sistemas (ERP como SAP o CRM).- Aplicaciones informáticas que imitan el funcionamiento de los sistemas guiándonos y corrigiéndonos en cada acción. Permiten poner a prueba los conocimientos y el manejo de los sistemas en 
$\underline{u n}$ entorno virtual antes de llevarlo a la práctica en el real. Pueden producirse con software como el i Tutor de SAP, el RoboDemo de Macromedia, y Assima de Educaterra.

- Simulación de entornos ( $\underline{\text { eer }}$ simuladores de vuelo, de guerra, de ciudades, de civilizaciones, etc.).- Juegos que se desarrollan en mundos virtuales, con tal nivel de detalle que los hacen muy similares a los reales. Algunos ejemplos de este tipo de software son el Sim City, el Fligth Simulator, etc. Estos juegos nos permiten diseñar, crear y administrar ciudades, o manejar aviones, autos y otras máquinas, a través de comandos, situaciones y sensaciones prácticamente reales. Los más simples pueden producirse con aplicaciones como el PowerSim.

- Simulación de situaciones o casos (de negociación, de management, etc.).Podemos encontrar juegos, casos o situaciones en las que se plantean problemas reales que deberán ser resueltos a partir de diversas opciones, tales como tableros de comando, aventuras gráficas, respuestas a preguntas, o selección de opciones. Se producen con aplicaciones especiales para animación en 3 D.

Un ejemplo que puede ilustrar mejor esta última categoría es el juego de simulación para el entrenamiento en la toma de decisiones gerenciales, desarrollado por el grupo Moar en España. El software simula el entorno de una oficina en la que el participante se convierte en el gerente de una compañía por un día. Es tal el nivel de detalle, que uno puede escuchar el teléfono de la oficina, entrar al baño a lavarse las manos o abrir una ventana como lo haría en la vida real. Durante esta virtual jornada laboral, el jugador debe tomar decisiones de distintos niveles de complejidad, que van desde la atención de la llamada de un cliente, el despido de personal o la realización de una millonaria operación financiera. En el transcurso del juego se presentan situaciones problemáticas que el participante deberá resolver en forma inmediata. Las decisiones tomadas durante el mismo determinarán el destino de la compañía.

La realidad virtual puede ser considerada como una forma $\underline{\underline{d e}}$ simulación que utiliza las tres dimensiones del espacio para brindar mayor realismo. Se utiliza frecuentemente en juegos y en situaciones donde se desea que el participante se sienta totalmente inmerso en el mundo simulado. Para ello se usa un casco con visión de $360^{\circ}$, y, en algunas 
ocasiones, guantes especiales para moverse y seleccionar las opciones del software. Esta tecnología es utilizada por arquitectos, ingenieros y militares, entre otros.

Las simulaciones constituyen excelentes herramientas para poner a prueba las competencias, motivar y proponer desafíos a los alumnos. Según explicó Elizabeth Gothelf en una entrevista concedida a la revista digital e-Learning América Latina ( $\left.\mathrm{N}^{\circ} 34\right)$, "problemas como la deserción o la falta de motivación de los estudiantes en entornos virtuales, podrían ser resueltos mediante el desarrollo de simulaciones capaces de consolidar el impulso educativo".

"Las simulaciones ayudan a incrementar la autenticidad del programa de aprendizaje, haciéndolo mas transmisible y motivador”, opina el especialista Marc. J. Rosemberg en su libro E-Learning: Estrategias para transmitir conocimiento en la Era Digital.

Asimismo, las simulaciones constituyen un componente más de una solución de eLearning y no pretenden reemplazar a otras estrategias de aprendizaje ni a la capacitación cara a cara. Estas ideas coinciden con las expuestas por el director de eLearning de Fycsa, Mariano García de la Fuente, y por el gerente de consultoría de Educaterra, Joaquín Azcue, entre otros expertos invitados a la mesa sobre simulaciones de On Line Educa Madrid 2004.

\section{Juegos Cooperativos}

Son aquellos centrados, más que en la competencia, en la promoción de la colaboración. Se utilizan para que las personas se conozcan e integren entre sí, conformando grupos, equipos de trabajo, de estudio o comunidades de aprendizaje.

Retener a los participantes hasta que completen sus estudios es una dificultad que enfrentan los entornos virtuales de aprendizaje. Las técnicas lúdicas, junto a las TICs, facilitan la creación y el mantenimiento de las comunidades virtuales de aprendizaje, intercambio y generación de información y conocimientos. Las técnicas lúdicas basadas en la cooperación promueven una dinámica que enriquece el proceso de aprendizaje grupal y facilita la construcción y la gestión del conocimiento compartido. Como dice un antiguo proverbio chino: "Ninguno de nosotros es tan inteligente como todos nosotros".

El concepto de "cognición repartida" se utiliza para definir el funcionamiento de la inteligencia humana a través de la colaboración en comunidades de aprendizaje, y su relación con diversos instrumentos. David Perkins lo define del siguiente modo: "Es la 


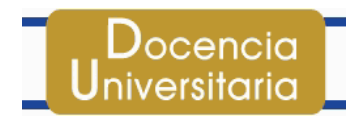

Aก̃ 3 - №1- Jun. 2007

dispersión del funcionamiento intelectual a través de instrumentos físicos, sociales y simbólicos (...) Normalmente, sea en sus hogares, en los lugares de trabajo o en la recreación, le gente funciona según distintas versiones de la persona más el entorno, haciendo uso intensivo de la información, los recursos físicos, y también de la acción y la dependencia con los otros". Entre los instrumentos que menciona David Perkins, destacan las tecnologías de la información y la comunicación. Para este autor, "la tecnología de la computación ha proporcionado una variedad de nuevos vehículos físicos para apoyar la cognición de los alumnos".

Algunas técnicas lúdicas para el pensamiento lateral fueron creadas y utilizadas durante años por Edward de Bono en el entrenamiento de ejecutivos. Con técnicas como Los Sombreros para Pensar, los participantes logran ponerse en el lugar de otros para observar y analizar un problema desde diversas perspectivas. Sigrid Loos considera que "la cooperación puede estimular el sentido de la comunidad (...) al brindar la posibilidad de cambiar el propio rol, tanto el asumido en el juego mismo como el que nos fue impuesto en el gran juego de la vida".

El juego cooperativo constituye por lo tanto una estrategia fundamental para el conocimiento, la integración y la conformación de comunidades de aprendizaje capaces de generar y compartir información, conocimiento y las mejores prácticas al interior de las organizaciones.

Los siguientes son algunos ejemplos de juegos cooperativos en entornos virtuales:

- $\quad$ Proyecto Atlas de la Diversidad Cultural: Juego del Gin Cana de la Diversidad (http://www.atlasdeladiversidad.net)

- Mi amiga vive lejos, pero me gusta hablar con ella: Juego virtual de conocimiento e integración. (http://www.miamiga.org)

- Desafío SEBRAE Argentina 2003: La Asociación de Dirigentes de Empresa (ADE) de Argentina, y el Servicio Brasileño de Apoyo a la Pequeña y Mediana Empresa (SEBRAE), lanzaron el este juego virtual de simulación empresaria para estudiantes de nivel universitario y terciario de instituciones públicas y privadas. (http://www.elearningamericalatina.com/edicion/junio2/it_6.php) 


\section{Del Juego Real al Virtual}

Las propuestas lúdicas que utilizan la palabra escrita o hablada y la imagen como componentes centrales, son ideales para su traslado a diferentes entornos virtuales de aprendizaje. Y para poder utilizar un juego del mundo real, solo es necesario adaptarlo al entorno y las herramientas tecnológicas con las que se va a implementar. El mail, el Chat, los foros, el Messenger, las aulas virtuales y los sistemas de audio y videoconferencia son los mejores instrumentos para trabajar con los recursos lúdicos.

Lo cierto es que algunas técnicas lúdicas del mundo real pueden potenciarse en entornos virtuales, mientras que otras deben ser transformadas para alcanzar el éxito. Para ilustrar esta transformación, utilizaremos el ejemplo del juego de la personalidad construida y dinámica que he aprovechado durante años en actividades cara a cara, y cuyo traslado a los entornos virtuales ha resultado sumamente exitoso.

\section{La personalidad construida}

Dinámica del juego cara a cara: Sentados en una ronda, cada participante escribe su nombre en una hoja y se la pasa al compañero de la derecha. El coordinador propone una frase incompleta que cada participante completará en función del nombre de la persona, escrito en la hoja recibida. A una señal suya, los jugadores vuelven a pasar las hojas en la misma dirección simultáneamente. El juego continúa con diferentes consignas hasta que cada miembro recupera su hoja. Para terminar la actividad, aquellos que lo deseen pueden leer en voz alta la descripción de su personalidad, construida por los diferentes integrantes del grupo.

Ejemplo de consignas:

- Juan es:

- Le gusta mucho:

- Sabe mucho de:

- Sueña con:

- Vive con:

- Es muy hábil para:

Funciones del juego: Conocer y expresar matices y características correspondientes a la personalidad y el aspecto físico de cada participante, según el punto de vista de los otros miembros del grupo. 


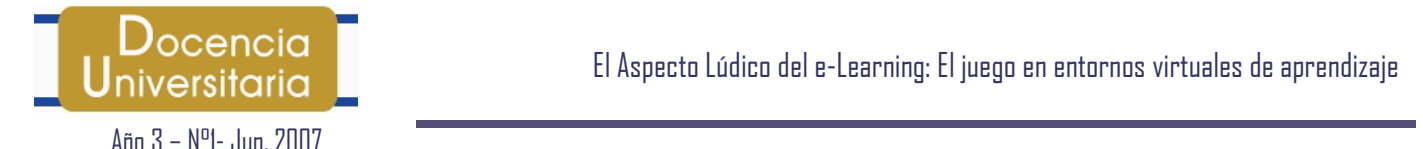

Dinámica lúdica en un entorno virtual: El juego se desarrolla respetando las mismas consignas y en base a una dinámica similar a la versión cara a cara. La ronda puede reemplazarse con un listado de participantes ordenados de manera tal que puedan enviarse e-mails en lugar de pasar la hoja.

Los mensajes de correo electrónico, que incluyen los nombres de los participantes en el Tema (Subject), se completan y reenvían al siguiente jugador de la lista para que agregue el texto correspondiente. Se puede utilizar un formulario de Word con las tareas escritas para que los participantes completen la primera actividad que encuentren vacía y la envíen al siguiente participante de su lista.

En un entorno virtual, la dinámica del juego cambia en función a los tiempos de respuesta, a la herramienta que se utilice, al perfil, y a la destreza y conocimiento de las personas que participen como tutores y alumnos.

\section{Para qué Sirve Jugar en un Entorno Virtual}

- Para facilitar la constitución del vínculo e integración del grupo (alumnos y docentes).

- Para motivar su participación y la expresión de toda su capacidad creativa.

- Para crear el clima propicio que facilite un buen proceso de aprendizaje.

- Para producir un modelo de enseñanza más creativo, entretenido, interesante e innovador.

- Para aumentar el grado de retención y el deseo de aprender a través de experiencias placenteras y emotivas.

- Para retener a los estudiantes por más tiempo, gracias a la consolidación de un grupo integrado y preparado para la tarea.

Naturalmente, para poder diseñar, coordinar o participar de dinámicas lúdicas cooperativas es necesario aprender a jugar abandonando los hábitos competitivos, conformando verdaderas comunidades de aprendizaje, intercambio, colaboración y generación de conocimientos. "Es una necesidad general del hombre sentirse parte de un grupo (...) y este es el primer paso para lograr su participación. El juego es un lenguaje y como tal permite que las personas se comuniquen y se unan entre sî́, afirma Sigrid Loos. 
Aก̃̃ 3 - №1- Jun. 2007

\section{Conclusiones}

En este capítulo hemos intentado $\underline{\text { hacer }}$ un recorrido por diferentes ideas, reflexiones y experiencias lúdicas virtuales. Espero que los lectores hayan disfrutado de la lectura y se animen a poner en práctica el juego en la producción e implementación de programas de educación virtual. Aprender a través de actividades lúdicas es una de las experiencias más placenteras, emotivas, y participativas que pueden tener nuestros alumnos, y representa un desafío para la creatividad de todos los que diseñamos y producimos estrategias de aprendizaje en el marco de los entornos virtuales.

Como dice Henry David Thoreau en el libro El Espíritu Creativo, de Daniel Goleman, "si avanzamos en la dirección de nuestros sueños, encontraremos un éxito inesperado en cualquier momento".

\section{Juego final}

Tal vez la mejor manera de ilustrar y terminar este artículo consista en proponer a los lectores a jugar con el texto. Para hacerlo, los invitamos a poner en práctica su capacidad lúdica buscando una última frase escondida. Para encontrarla, deberán hallar en este capítulo las palabras resaltadas con negrita, cursiva y subrayado, y ordenarlas de manera tal que puedan construir una frase coherente y estrechamente relacionada con la filosofía planteada. El resultado de esta actividad será presentado al final en una posición diferente, de modo tal que para leerlo tengan que girar el libro.

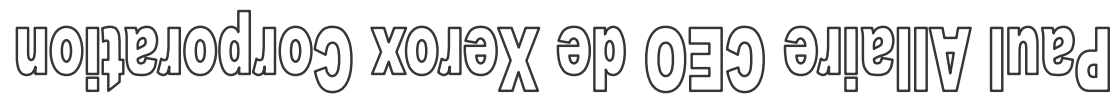

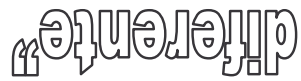

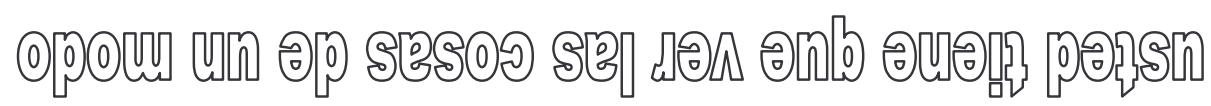

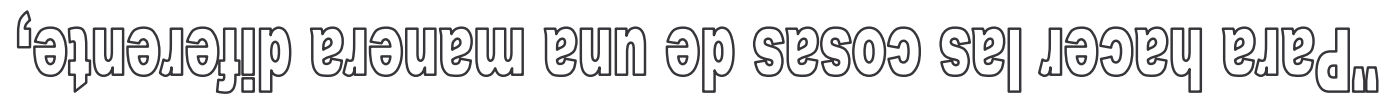




\section{Bibliografía}

ABERASTURY, Arminda

1968 El Niño y sus Juegos. Buenos Aires: Paidós

DE BONO, Edward

1994 El Pensamiento Creativo. Barcelona: Paidós

GARDNER, Howard

1993 La Mente No Escolarizada. Barcelona: Paidós

GOLEMAN, Daniel

1999 La Inteligencia Emocional. Buenos Aires: Vergara

GOLEMAN, Daniel, KAUFMAN, Paul y RAY, Michael

2000 El Espíritu Creativo. Buenos Aires: Vergara

HUIZINGA, Johan

1968 Homo Ludens. ¿CIUDAD?: Emecé

LOOS, Sigrid

1989 Noventanove Giochi Cooperativi. Torino: Gruppo Abele

PERKINS, David

1995 La Escuela Inteligente. Barcelona: Gedisa

ROSEMBERG, Marc

2001 E-Learning: Estrategias para transmitir conocimiento en la Era Digital. Bogotá: Mc Graw Hill 
Aก̃ 3 - Nㅣ- ل」un. 2007

Para citar este documento, puede utilizar la siguiente referencia:

MARGUILIS, Lucio (2007). "El Aspecto Lúdico del e-learning; El juego en entorrnos virtuales de aprendizaje".

[artículo en línea]. Revista Digital de Investigación en Docencia Universitaria (RIDU) Año 3 - $N^{\circ}$ 1-Junio 2007.

[Fecha de consulta: dd/mm/aa]. <http://beta.upc.edu.pe/calidadeducativa/ridu/2007/ridu3 5LM.pdf $>>$

\section{Lucio Marguilis}

1margulis@yahoo.com

Especialista en Educación con experiencia en diseño, desarrollo y ejecución de cursos, y proyectos de capacitación en empresas e instituciones internacionales. Ha dictado cursos y desarrollado proyectos de capacitación presencial y utilizando soluciones de e-Learning y Change Management a través de Técnicas Lúdicas en el Joint (American Jewish Joint Distribution Committee), la Sociedad Italiana para la Educación Musical, la Sociedad de la Academia de Lombardía, el Ministerio del Trabajo de Italia, la Universidad de Flores de Argentina, la Universidad Tecnológica Nacional, el Fondo Social Europeo y la UNED (Universidad Nacional de Educación a Distancia, España).

Docente del módulo de Elaboración de Contenidos Formativos del Master en Dirección de Proyectos de e-Learning de la Universidad Politécnica de Madrid. Del curso sobre juegos para el aprendizaje Técnicas y Tácticas para Aprender Jugando de la Asociación de e-Learning de España AEFOL, y del curso sobre Nuevas Metodologías Alternativas de Enseñanza: Integración al Aula y su Aplicación en el Proceso de Aprendizaje de las Nuevas Generaciones de la Universidad Virtual de la Caja Rural de Toledo, entre otros. 